\title{
Noninvasive Bioreactance-Based Fluid Management Monitoring: A Review of Literature
}

\author{
Yatin Mehta ${ }^{1}$ Poonam Malhotra Kapoor ${ }^{2}$ Harish Mallapura Maheswarappa ${ }^{3}$ Gaurav Saxena ${ }^{4}$
}

${ }^{1}$ Medanta Institute of Critical Care and Anesthesiology, Medanta the Medicity, Gurugram, Haryana, India

2 Department of Cardiac Anesthesiology, All India Institute of Medical Sciences, New Delhi, India

${ }^{3}$ Division of Critical Care Medicine, Critical Care and Pain, Department of Anaesthesiology, Tata Memorial Hospital, Mumbai, Maharashtra, India

${ }^{4}$ Medical Affairs Division, Baxter India Pvt Ltd, Gurugram, Haryana, India

\author{
Address for correspondence Gaurav Saxena, MBBS, Medical Affairs, \\ Baxter India Pvt Ltd, Gurugram, Haryana, 122002, India \\ (e-mail: Gaurav_saxena@baxter.com).
}

\begin{abstract}
Body fluid balance is an independent predictor of mortality. For each liter of fluid over and above $5 \mathrm{~L}$, risk-adjusted excess mortality is seen. Mortality increased by $2.3 \%$ for each $1 \mathrm{~L}$ of fluid and hospital costs increased by $\$ 999$. Accordingly, most recent guidelines have endorsed dynamic modeling. Passive leg raising-induced increase of aortic blood flow $\geq 10 \%$ predicts fluid responsiveness with a sensitivity of $97 \%$ and a specificity of $94 \%$. Thus, passive leg raising is often used as gold standard for validation of other procedures (though it's usefulness to assess respiratory variation in vena cava is not conclusive). STARLING, a device based on bioreactance, works on phase shift or time delay while bioimpedance works on the amplitude of the thoracic impedance. Unlike bioimpedance, bioreactance is not affected by the size of the patient, thoracic fluids, or position of sensors.

STARLING is equipped with four sensor pads. Each pad contains two sensors, the outer sensor is a transmitting electrode and the inner sensor is a receiving electrode. The

Keywords

- bioreactance technology

- noninvasive fluid management

- STARLING STARLING monitor induces a 75-KHz AC current. It then measures the time delay/phase shift.

STARLING system, a bioreactance-based dynamic assessment system for fluid responsiveness, predicts it accurately, precisely, and noninvasively. It reduces invasive risks and is independently validated against pulmonary artery catheter. It is not affected by vasopressors or shock and has wide range of application.
\end{abstract}

\section{Introduction}

Body fluid balance is an independent predictor of mortality. This is even more relevant in context of replenishment of fluid in conditions like shock-wherein less or more replen- ishment is equally hazardous. To depict it another way, the fluid-volume versus complication curve can be plotted in Ushape, with minima in the middle physiological region as shown in - Fig. 1.
DOI https://doi.org/ 10.1055/s-0041-1741491. ISSN 2457-0206. (c) 2022. Official Publication of The Simulation Society (TSS), accredited by International Society of Cardiovascular Ultrasound (ISCU). All rights reserved.

This is an open access article published by Thieme under the terms of the Creative Commons Attribution-NonDerivative-NonCommercial-License, permitting copying and reproduction so long as the original work is given appropriate credit. Contents may not be used for commercial purposes, or adapted, remixed, transformed or built upon. (https://creativecommons.org/ licenses/by-nc-nd/4.0/)

Thieme Medical and Scientific Publishers Pvt. Ltd., A-12, 2nd Floor, Sector 2, Noida-201301 UP, India 


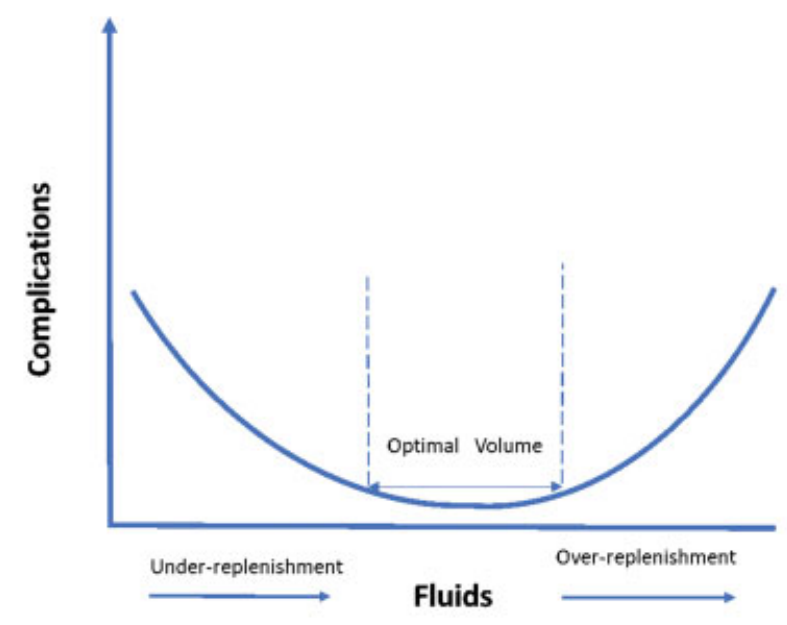

Fig. 1 Fluid volume versus complication curve. ${ }^{1}$

At the same time, it is also known that in critical conditions, body fluids function through volume expansion and in addition to their actions as a matrix of various metabolic activities, they also act as the transport vehicle of nutrition, oxygenation, and excretion. Thereby, the replenished fluid is contextually a drug with low therapeutic index (analogous to antiarrhythmic drug which lead to arrhythmia in absence as well as when in excess) (-Fig. 2).

The study was performed upon 23,513 severe sepsis/septic shock cases admitted to intensive care unit (ICU) and for each liter of fluid over and above $5 \mathrm{~L}$, riskadjusted excess mortality is seen. Mortality increased by 2.3\% (95\% confidence interval $2.0-2.5 \%, p=0.005$ ) for each $1 \mathrm{~L}$ of fluid and hospital costs increased by $\$ 999 .^{2}$ Thus, the propensity model analysis looking at "mortality and fluid administration" using age and "acute conditions on admission" supports the hypothesis that fluid balance is an independent predictor of mortality.

Recently, researches have concluded that "considering the changing adult cardiac surgery patient population and continuous advances in health care, dynamic models are preferred over static models for assessing volume responsiveness of a patient in shock." ${ }^{3}$ Accordingly, most recent guidelines, namely National Institute of Health and Clinical Excellence (U.K.) (2011), SFAR (French Society of Anaesthesia and Intensive Care Medicine; France) (2013), European Society of Intensive Care Medicine (2014), DGAI (German Society of Anaesthesiology and Intensive Care Medicine; Germany) (2014), National Quality Forum (2015), and Society of Critical Care Medicine (SCCM 2015) Surviving Sepsis Campaign (SSC 2013, 2017) have endorsed dynamic modeling.

Against the passive leg raising (PLR) and trending $500 \mathrm{~mL}$ fluid challenge, a minor bolus challenge of 300 was compared for assessing the fluid responsiveness-all the methods imply a play of Frank-Starling law. PLR-induced changes in cardiac output $(\mathrm{CO})$ reliably predict the response of $\mathrm{CO}$ to volume expansion in adults with acute circulatory failure. PLR-induced increase of aortic blood flow $\geq 10 \%$ predicts fluid responsiveness with a sensitivity of $97 \%$ and a specificity of $94 \% .{ }^{4}$

The question is that "do all hemodynamically unstable patients respond to a bolus of intravenous fluids-nearly half will not respond to intravenous bolus?" In a meta-analysis of 50 ICU studies and 2,260 patients, only half of patients were fluid responsive (at 95\% confidence interval, $42-56 \%$, with a sensitivity of $88 \%$, and specificity of $92 \%){ }^{4}$

This fact may manifest because it is also a fact that on the flatter right extreme of the Frank-Starling curve, the same

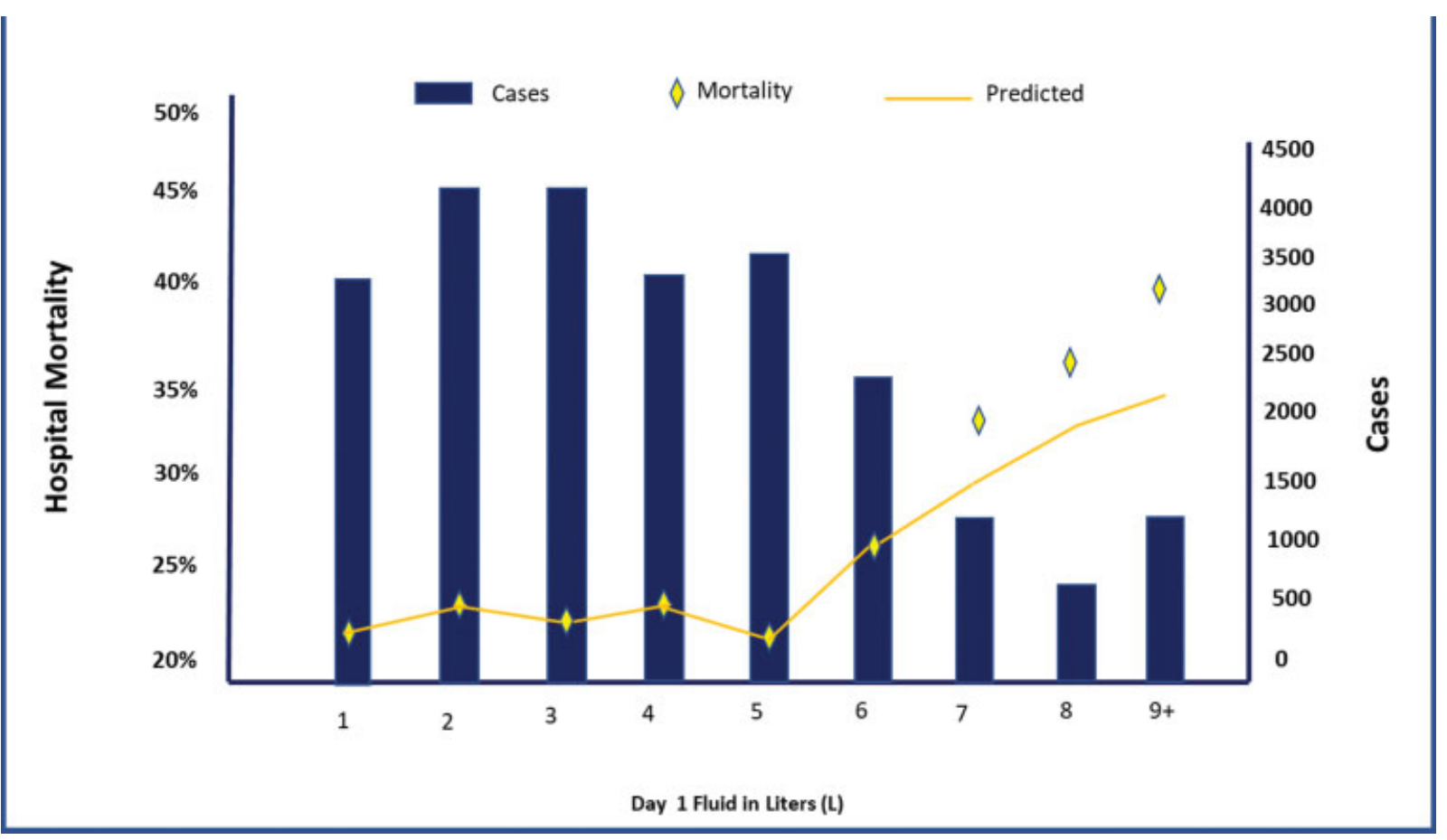

Fig. 2 Daily fluid replenishment and occurrence of complications. ${ }^{2}$ 


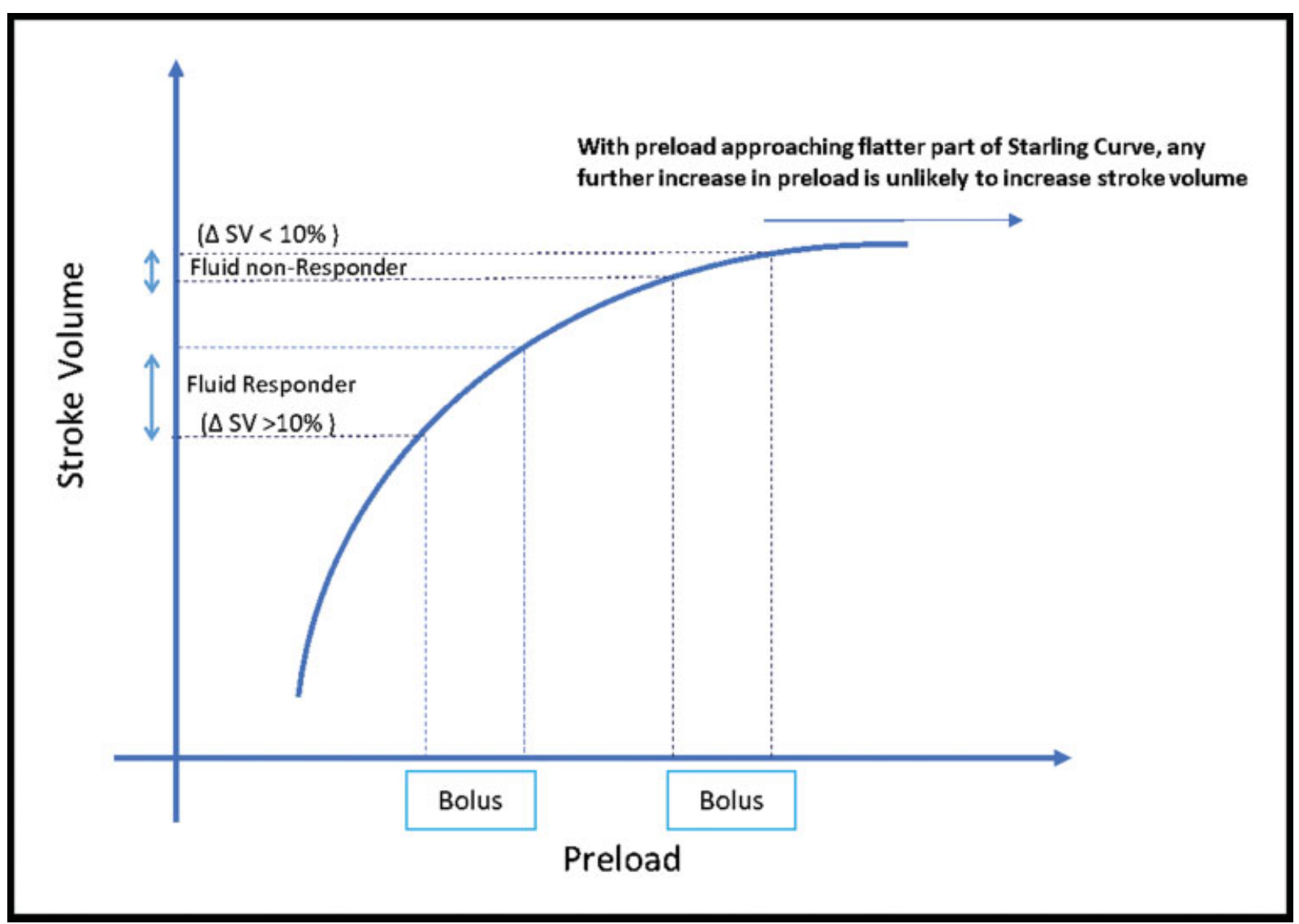

Fig. 3 Refractory (or less responsive) stroke volume on fluid responsiveness test (adapted from Cecconi M, Parsons AK, Rhodes A. What is a fluid challenge? ${ }^{5}$

amount of the bolus fluid is not as effective in increasing the stroke volume as at the steeper part of the curve (-Fig. 3).

Moreover, when PLR effects are assessed by changes in pulse pressure, the specificity of the PLR test remains acceptable, but its sensitivity is poor. Still PLR followed by measurement of $\mathrm{CO}$ or related parameters may be the most useful tests for predicting fluid responsiveness in hemodynamically unstable adults. ${ }^{4,6}$ Although the usefulness in assessment of respiratory variation in the vena cava requires confirmatory studies. $^{4}$

Other commonly used CO measurement methods include indirect Fick methods, thermodilution (TD), Doppler ultrasound or echocardiography, partial carbon dioxide rebreathing, thoracic electrical bioimpedance, and magnetic resonance imaging. TD via the pulmonary artery catheter
(PAC) is still considered to be the gold standard method in the clinical setting. ${ }^{7}$

Coming to the inherent technological difference, a device based on bioreactance (Starling, Baxter Inc. Deerfield, United States) works on phase shift or time delay (-Fig. 4A), while bioimpedance works on the amplitude of the thoracic impedance. The flow of blood in the thorax introduces a time delay or "phase shift" in STARLING signal. These signal changes have been further correlated to the known TD CO of 65,000 patient samples in multiple clinical settings.

Unlike bioimpedance, bioreactance is not affected by the size of the patient, thoracic fluids (e.g., pleural effusion/pulmonary edema), or position of sensors. And here lies the advantage of a device based on bioreactance if utility and cost-effectiveness parameters are acceptable.
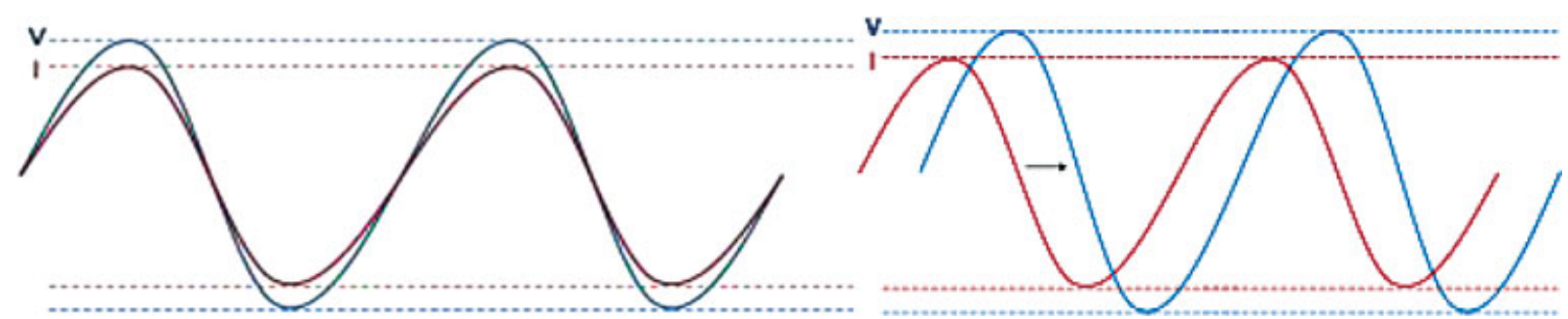

Fig. 4 Parameter measured in bioimpedance (A: left) versus bioreactance (B: right). 


\section{Method}

We searched Scholar, MEDLINE, Embase, and the Cochrane Central Register of Controlled Trials (CENTRAL) in the COCHRANE Library. The Medical Subject Headings terms "Fluid Management," "Fluid responsiveness," and "Non Invasive fluid management monitoring," "Bio-Reactance," "STARLING" were used. Additional studies were identified by review of the reference sections of all eligible studies. The aim of this review article is to bring together the evidences with bioreactance-based fluid management monitoring in critically ill patients.

\section{STARLING}

The STARLING system ( - Fig. 5) is equipped with four sensor pads. Each pad contains two sensors, the outer sensor is a transmitting electrode and the inner sensor is a receiving

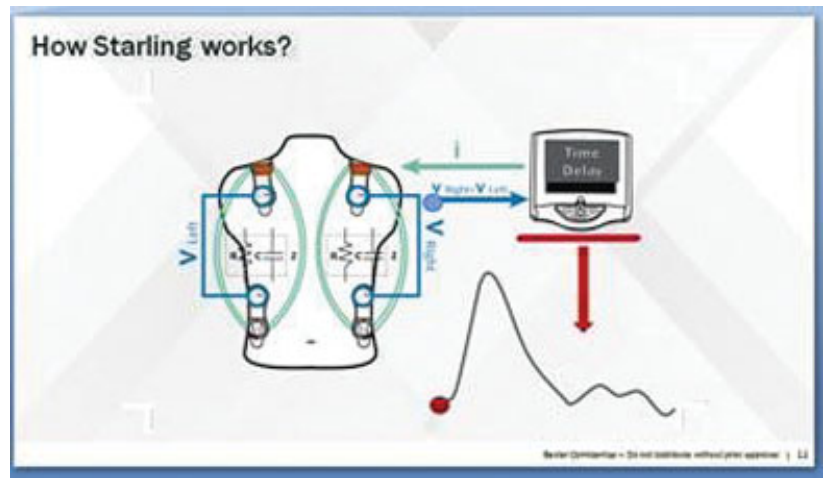

Fig. 5 STARLING: working principle (phase shift). electrode. The sensors are applied around the heart on the chest or back (there is no need for exact positioning).

The STARLING monitor induces a $75 \mathrm{KHz}$ AC current to the thorax via the outer sensors and receives the voltage via the inner sensors. It then measures the time delay/phase shift (as depicted in $\mathbf{F i g}$. $\mathbf{4 A}$ ) between the induced current and the received voltage continuously, thus building the STARLING signal. The final mechanistic flowchart is as shown in - Fig. 6.

\section{STARLING Validation Data with FloTrac, Esophageal Doppler Monitoring, and Thermodilution}

CO measurements obtained from bioreactance-based monitoring and TD were simultaneously recorded minute-byminute and compared in 110 patients. Bioreactance-based monitoring was evaluated for the accuracy, precision, responsiveness, and reliability for detecting $\mathrm{CO}$ changes. Total of 65,888 pairs of $\mathrm{CO}$ measurements were collected from 110 patients. $^{8}$

In this study, ${ }^{7}$ the authors concluded that bioreactancebased fluid monitoring system had acceptable accuracy, precision, and responsiveness for $\mathrm{CO}$ monitoring in patients experiencing a wide range of circulatory situations. In yet another study, ${ }^{9}$ it was found that $\mathrm{CO}$ measured via bioreactance is precise and reliably measures $\mathrm{CO}$ at rest and changes in $\mathrm{CO}$ with vasodilator challenge in patients with pulmonary hypertension.

Bioreactance and Esophageal Doppler Monitor in Guiding Goal-Directed Fluid Therapy

Using McNemar's $p$-value and 95\% of agreement limit, a study ${ }^{10}$ has shown that the noninvasive CO monitoring performs similarly to the esophageal Doppler monitor in

\section{STARLING STROKE VOLUME}

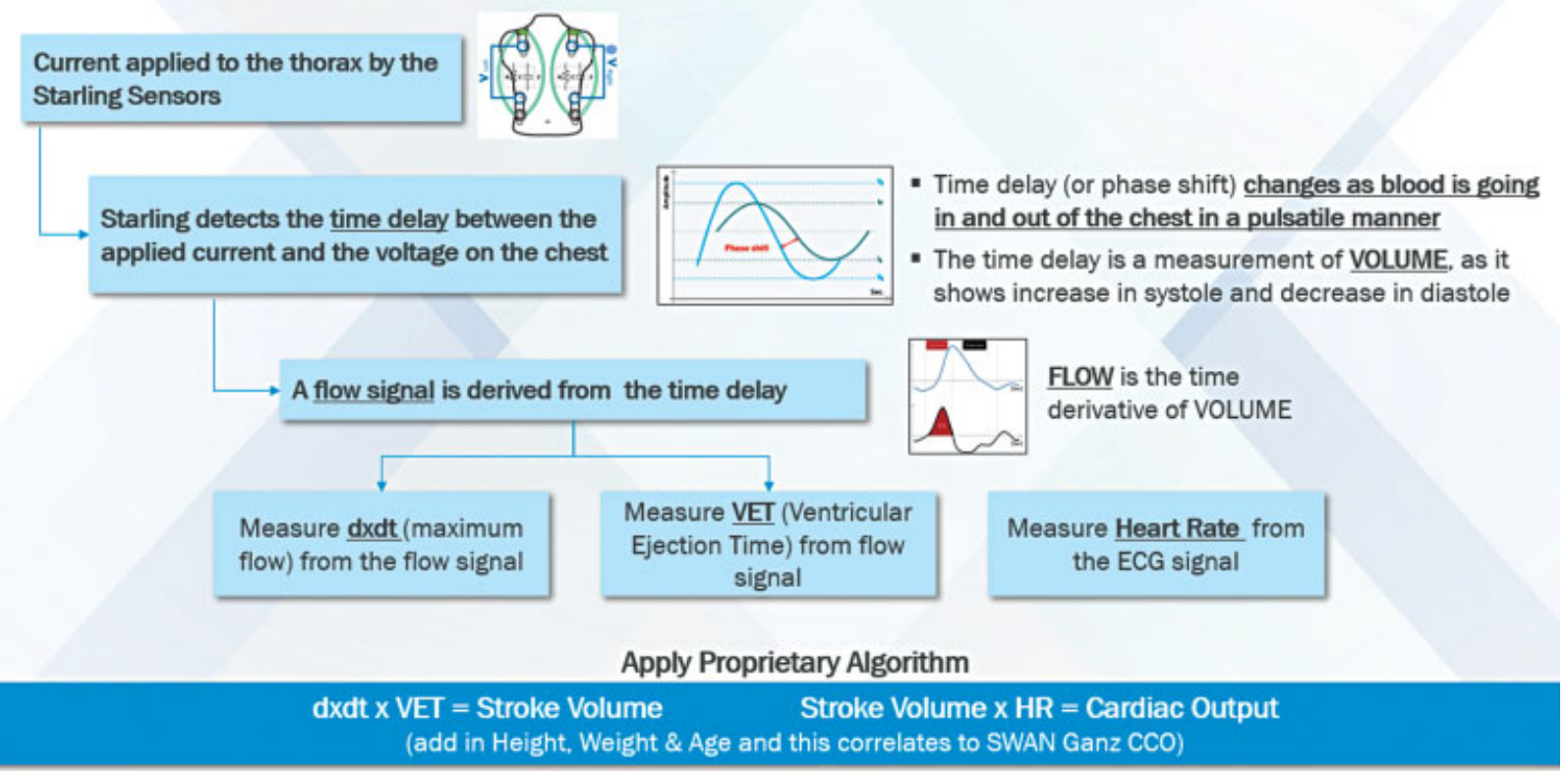

Fig. 6 STARLING: A noninvasive fluid management monitoring system. 
guiding goal-directed fluid therapy, with no clinically significant differences in outcomes, and offers increased ease of use as well as fewer missing data points. Thus, the former may be a viable alternative monitor to guide.

This study was conducted in two consecutive phases. Phase I was conducted over an 8-month period, whereas phase II was conducted over a 6-month period immediately after phase I. Phase I and phase II patients had similar clinical outcomes, with no clinically significant differences in length of hospital stay, postoperative bowel function recovery, renal function, or other complications.

In phase I (50 patients), investigators used the esophageal Doppler monitor in guiding goal-directed fluid therapy while the bioreactance-based fluid monitoring was also connected and recorded hemodynamic variables. All decisions to administer fluid during phase I were based on measurements from the esophageal Doppler monitor, with no consideration given to the bioreactance-based fluid monitoring measurements.

In phase II (50 patients), goal-directed fluid therapy was guided by the bioreactance-based fluid monitoring measurements while hemodynamic variables were simultaneously recorded by the esophageal Doppler monitor. All decisions to administer fluid during phase II were based on measurements from the bioreactance-based fluid monitoring, with no consideration given to the esophageal Doppler monitor measurements.

There was reasonable correlation in baseline stroke volume. There also was agreement on fluid responsiveness between the monitors $66 \%$ of times and estimated to be at least $61 \%$ at 15 minutes with no significant systematic disagreement at any time point. In the study, ${ }^{10}$ no significant differences were found:

1. Between the bioreactance-based fluid monitoring and esophageal Doppler monitor in ability to assess fluid responsiveness as part of a goal-directed fluid therapy algorithm.

2. In length of hospital stay and postoperative recovery when goal-directed fluid therapy was guided by the bioreactance-based fluid monitoring or the esophageal Doppler monitor.

Benefits of Noninvasive Fluid Response Monitoring in Indian Patients as Compared with Invasive (PAC) or Minimally Invasive (Vigileo, Transesophageal Echocardiography) (Consensus) Methods In a study ${ }^{11}$ of consecutive 29 patients, 12,099 simultaneous reading with three devices was conducted. TD-based continuous CO monitoring with PAC following cardiac surgery as gold standard was also done along with the two other devices, viz. minimally invasive FloTrac-Vigileo and a totally noninvasive $\mathrm{CO}$ monitoring.

$\mathrm{CO}$ values obtained simultaneously by the three systems were recorded continuously on a minute-by-minute basis. The new technology of noninvasive CO monitoring was considered as:

- Acceptably accurate when bias of measurement was less than $20 \%$; acceptably precise when random error of measurements around the mean value were less than 20\%; acceptably responsive when time delay and amplitude of change were at least equivalent to PAC determined continuous CO.

- Acceptably reliable when specificity and sensitivity in detecting simultaneous directional changes in $\mathrm{CO}$ was close to one.

\section{Kansas University Trial}

In this study, ${ }^{12}$ dynamic measure (stroke volume changes) used to guide fluid therapy in septic shock was associated with better clinical and economic outcomes. To determine whether stroke volume-guided fluid resuscitation in patients with severe sepsis and septic shock alters ICU fluid balance and secondary outcomes, this retrospective cohort study evaluated consecutive patients admitted to an ICU with the primary diagnosis of severe sepsis or septic shock. Cohorts were based on fluid resuscitation guided by changes in stroke volume or by usual care.

\section{Fluid Response Evaluation in Sepsis Hypotension and Shock (FRESH) Study}

In a prospective, multicenter, randomized clinical trial at 13 hospitals in the United States and United Kingdom, ${ }^{13}$ patients presented to emergency departments with sepsis that was associated with hypotension and anticipated ICU admission were studied. Intervention arm patients were assessed for fluid responsiveness before clinically driven fluid bolus or increase in vasopressors requirement.

The protocol included reassessment and therapy as indicated by the PLR result. The control arm received usual care. The primary clinical outcome was positive fluid balance at 72 hours or ICU discharge, whichever occurred first. Stroke volume-guided fluid and vasopressor management was used continuously during the intervention period ( 72 hours or ICU discharge, whichever occurred first).

An increase in stroke volume $\geq 10 \%$ was considered fluid responsiveness. If the patient demonstrated fluid responsiveness, protocol prompts were provided to administer a crystalloid fluid bolus $(500 \mathrm{~mL})$ for persistent hypotension, with repeat PLRs after every fluid bolus.

If the patient was not fluid responsive, the initiation or up-titration of vasopressors was prompted with repeat PLR after significant escalation (an increase of $1 \mu \mathrm{g} / \mathrm{kg} / \mathrm{min}$ norepinephrine). In this manner, the protocol allowed for the physiologic titration of both fluid and vasopressors to treat hypoperfusion.

The volume of administered fluids at 72 hours was significantly less in patients in the intervention arm than the usual care arm. Separation between arms for 72-hour fluid balance and total amount of administered intravenous fluids were maintained even when preenrollment fluid was included in the analysis.

Consistent with current guideline recommendations, patients received 2 to $3 \mathrm{~L}$ of intravenous fluids before randomization in both arms. These results indicate that PLRguided protocol instructions during the first 72 hours of care accounted for the observed differences in fluid balance 
between arms. There was a reduced need for renal replacement therapy or invasive mechanical ventilation in the intervention arm.

On pre-post intervention assessment, a larger fluid difference $(3.59 \mathrm{~L})$ was reported between patients who were treated with PLR-guided strategy and usual care. Though authors found a smaller difference in the modified intent-totreat analysis of the Fluid Response Evaluation in Sepsis Hypotension and Shock (FRESH) study, it can be due to more restrictive fluid management in the usual care arm.

However, the concordance in a lower risk for renal and respiratory failure between the two arms in the two studies suggests a consistent and strong clinical effect.

Additionally, studies of dynamic measure-guided fluid administration in surgical patients have reported comparable overall fluid administration between dynamic measureguided treatment arms and usual care arms with greater preservation of renal and respiratory function in the intervention arm.

\section{STARLING: Implementing Noninvasive Fluid Management Monitoring in Indian Setting}

Because of the limitations of bioimpedance devices, newer methods of processing the impedance signal have been developed. Human thorax and thoracic content is reason for their resistance $(\mathrm{R})$ and capacitance $(\mathrm{C})$, which together create the thoracic impedance (Zo), when a current is passed through thorax. Phase shifts can occur only because of pulsatile flow. The overwhelming majority of thoracic pulsatile flow stems from the aorta. Therefore, the STARLING signal is correlated almost wholly with aortic flow. Furthermore, because the underlying level of thoracic fluid is relatively static, neither the underlying levels of thoracic fluids nor their changes induce any phase shifts and do not contribute to its signal. ${ }^{14}$

The $\mathrm{CO}$ as measured by bioreactance has been shown to highly correlate with that measured by TD and pulse contour analysis. ${ }^{15-19}$ Squara et a ${ }^{16}$ compared the STARLING system with PAC-derived $\mathrm{CO}$ in 110 patients after cardiac surgery. The reported bias was $\pm 0.16 \mathrm{~L} / \mathrm{min}$; Limits of Agreement (LOA) on Bland and Altman representation of $\pm 1.04 \mathrm{~L} / \mathrm{min}$ with a relative error of $9 \%$. The precision of the STARLING system was better than that of TD, with the device being able to track changes in $\mathrm{CO}$ accurately.

In a study of three ICUs (70 patients), Raval et $\mathrm{al}^{15}$ reported a bias of $-0.09 \mathrm{~L} / \mathrm{min}$ and an LOA of $\pm 2.4 \mathrm{~L} / \mathrm{min}$, with the STARLING system closely tracking changes in the TD CO. Rich et $\mathrm{al}^{17}$ performed right-heart catheterization in 24 patients with pulmonary hypertension. Simultaneous CO measurements were performed using TD, STARLING, and the Fick methods at baseline and after adenosine vasodilator challenge. CO measured by the STARLING system was significantly more precise than that of TD. The adenosine challenge resulted in a similar mean increase in $\mathrm{CO}$ with each method. The accuracy of STARLING was assessed in hemodynamically unstable ICU patients and healthy volunteers after PLR and fluid challenges using carotid and brachial arterial Doppler ultrasound flow $(\mathrm{mL} / \mathrm{min})$ as the reference technique and confirmed almost same as in a previous study ${ }^{19,20}$ where almost $100 \%$ concordance was found between fluid responsiveness as determined by carotid flow and the NICOM system.

Benomar et $\mathrm{al}^{21}$ showed that the NICOM system could predict fluid responsiveness accurately from changes in $\mathrm{CO}$ during PLR. As part of goal-directed perioperative therapy in patients undergoing major surgery, Waldron et $\mathrm{al}^{22}$ compared fluid responsiveness with an esophageal Doppler monitor and the NICOM system. Notwithstanding the limitations of esophageal Doppler monitoring (as discussed previously), there was a good agreement between these technologies.

STARLING system, personalized for fluid therapy:

1. Provides a dynamic assessment of fluid responsivenessaccurately, precisely, and $100 \%$ noninvasively. It supports individualized fluid therapy without requiring an invasive arterial or central line. It may reduce the risk of hospitalacquired infections and other complications. It has been independently validated against PAC, considered to be current gold standard in fluid monitoring therapy. Accuracy not affected by vasopressors or shock state and works in mechanically ventilated and spontaneously breathing patient. In Kansas trial also discussed previously, a retrospective analysis ${ }^{11}$ of 200 sepsis/septic shock patient has shown a cost-benefit of $\$ 14,498$ per patient as result of reduction in ICU length of stay, acute dialysis, and mechanical ventilation. Although this was the study conducted in United States, its implication may extend to developing countries with significant health care burden like India. In view of high recurring costs associated with other invasive or minimally invasive hemodynamic monitoring modalities for the disposables, noninvasive disposable sensor pads used for STARLING can further reduce the recurring cost and economic burden of Indian patient.

STARLING system seems to be accurate, precise, and reasonably validated technology with over 500 patients in published clinical studies. Multiple clinical settings (ICU/operating room/emergency department/exercise laboratory/out of hospital) against all major technologies (Swan Ganz, Pulse Contour, Doppler, Fick) with over 100 peer-reviewed publications.

\section{Conclusion}

Overall, given the clinical apropos, economic benefits with STARLING-based stroke volume-guided fluid therapy, its impact on current clinical practice would mostly rely upon the advantage of noninvasiveness (and consequential benefits like less chances of procedural cost/pain/infection/hospital stay, etc.) and wide spectrum of patient applicability.

\section{Conflict of Interest}

The corresponding author is an Employee of Baxter India Pvt Ltd. No conflict for the remaining authors.

\section{References}

1 Bellamy MC. Wet, dry or something else? Br J Anaesth 2006;97 (06):755-757 
2 Marik PE, Linde-Zwirble WT, Bittner EA, Sahatjian J, Hansell D. Fluid administration in severe sepsis and septic shock, patterns and outcomes: an analysis of a large national database. Intensive Care Med 2017;43(05):625-632

3 Siregar S, Nieboer D, Vergouwe Y, et al. Improved prediction by dynamic modeling: an exploratory study in the Adult Cardiac Surgery database of the Netherlands Association for Cardio-Thoracic Surgery. Circ Cardiovasc Qual Outcomes 2016;9(02):171-181

4 Bentzer P, Griesdale DE, Boyd J, MacLean K, Sirounis D, Ayas NT. Will this hemodynamically unstable patient respond to a bolus of intravenous fluids? JAMA 2016;316(12):1298-1309

5 Cecconi M, Parsons AK, Rhodes A. What is a fluid challenge? Curr Opin Crit Care 2011;17(03):290-295

6 Monnet X, Teboul JL. Passive leg raising for predicting fluid responsiveness: a systematic review and meta-analysis. Intensive Care Med Exp 2015;3(S):A587

7 Zhang Y, Wang Y, Shi J, Hua Z, Xu J. Cardiac output measurements via echocardiography versus thermodilution: a systematic review and meta-analysis. PLoS One 2019;14(10):e0222105

8 Squara P, Denjean D, Estagnasie P, Brusset A, Dib JC, Dubois C. Noninvasive cardiac output monitoring (NICOM): a clinical validation. Intensive Care Med 2007;33(07):1191-1194

9 Rich JD, Archer SL, Rich S. Noninvasive cardiac output measurements in patients with pulmonary hypertension. Eur Respir J 2013;42(01):125-133

10 Waldron NH, Miller TE, Thacker JK, et al. A prospective comparison of a noninvasive cardiac output monitor versus esophageal Doppler monitor for goal-directed fluid therapy in colorectal surgery patients. Anesth Analg 2014;118(05):966-975

11 Marqué S, Cariou A, Chiche JD, Squara P. Comparison between Flotrac-Vigileo and Bioreactance, a totally noninvasive method for cardiac output monitoring. Crit Care 2009;13(03):R73

12 Latham HE, Bengtson CD, Satterwhite L, et al. Stroke volume guided resuscitation in severe sepsis and septic shock improves outcomes. J Crit Care 2017;42:42-46
13 Douglas IS, Alapat PM, Corl KA, et al. Fluid response evaluation in sepsis hypotension and shock: a randomized clinical trial. Chest 2020;158(04):1431-1445

14 Keren H, Burkhoff D, Squara P. Evaluation of a noninvasive continuous cardiac output monitoring system based on thoracic bioreactance. Am J Physiol Heart Circ Physiol 2007;293(01): H583-H589

15 Raval NY, Squara P, Cleman M, Yalamanchili K, Winklmaier M, Burkhoff D. Multicenter evaluation of noninvasive cardiac output measurement by bioreactance technique. J Clin Monit Comput 2008;22(02):113-119

16 Squara P, Rotcajg D, Denjean D, Estagnasie P, Brusset A. Comparison of monitoring performance of Bioreactance vs. pulse contour during lung recruitment maneuvers. Crit Care 2009;13(04):R125

17 Rich JD, Archer SL, Rich S. Evaluation of noninvasively measured cardiac output in patients with pulmonary hypertension. Am J Respir Crit Care Med 2011;183:A6440

18 Heerdt PM, Wagner CL, DeMais M, Savarese JJ. Noninvasive cardiac output monitoring with bioreactance as an alternative to invasive instrumentation for preclinical drug evaluation in beagles. J Pharmacol Toxicol Methods 2011;64(02): 111-118

19 Marik PE. Noninvasive cardiac output monitors: a state-of the-art review. J Cardiothorac Vasc Anesth 2013;27(01):121-134

20 Marik PE, Levitov A, Young A, et al. The use of NICOM (bioreactance) and carotid Doppler to determine volume responsiveness and blood flow redistribution following passive leg raising in hemodynamically unstable patients. Ann Crit Care 2012

21 Benomar B, Ouattara A, Estagnasie P, Brusset A, Squara P. Fluid responsiveness predicted by noninvasive bioreactance-based passive leg raise test. Intensive Care Med 2010;36(11): $1875-1881$

22 Waldron NH, Miller TE, Nardiello J, et al. NICOM versus EDM guided goal directed fluid therapy in the perioperative period. Anesthesiology 2011;115:A680 